\title{
RESOLUTION-PRESERVING SPECKLE REDUCTION OF SAR IMAGES: THE BENEFITS OF SPECKLE DECORRELATION AND TARGETS EXTRACTION
}

\author{
Rémy Abergel ${ }^{1}$, Lö̈c Denis ${ }^{2}$, Florence Tupin $^{3}$, Saïd Ladjal ${ }^{3}$, Charles-Alban Deledalle $^{4}$, Andrés Almansa ${ }^{1}$ \\ ${ }^{1}$ Laboratoire MAP5 (CNRS UMR 8145), Université Paris Descartes, Sorbonne Paris Cité, France \\ ${ }^{2}$ Univ Lyon, UJM-Saint-Etienne, Institut d'Optique Graduate School, Laboratoire Hubert Curien CNRS UMR 5516, Saint-Etienne, France \\ ${ }^{3}$ LTCI, Télécom ParisTech, Université Paris Saclay, Paris, France \\ ${ }^{4}$ IMB, CNRS, Univ. Bordeaux, Bordeaux INP, F-33405 Talence, France
}

\begin{abstract}
Speckle reduction is a necessary step for many applications. Very effective methods have been developed in the recent years for singleimage speckle reduction and multi-temporal speckle filtering. However, to reduce the presence of sidelobes around bright targets, SAR images are spectrally weighted and this processing impacts the speckle statistics by introducing spatial correlations. These correlations severely impact speckle reduction methods that require uncorrelated speckle as input. Thus, spatial down-sampling is typically applied to reduce the speckle spatial correlations prior to speckle filtering. To better preserve the spatial resolution, we describe how to correctly resample SAR images and extract bright targets in order to process full-resolution images with speckle-reduction methods.
\end{abstract}

Index Terms - Sentinel-1, deramping, sub-pixel target detection, sidelobe reduction, despeckling.

\section{INTRODUCTION}

The analysis of SAR images requires a speckle reduction step. While this step was for a long time performed by local averaging (spatial multi-looking), with the recent progress accomplished in speckle filtering the use of more evolved methods cannot be overlooked. The goal of speckle reduction methods is to suppress as much as possible the speckle fluctuations while preserving at best the spatial resolution (i.e., without introducing notable blurring). To achieve this goal, the methods combine observed SAR intensities based on transforms (e.g., wavelets transforms), image models (e.g., total variation minimization, sparse coding), selection approaches (e.g., the sigma filter, patch comparisons) or learned transforms (e.g., deep neural networks). In order to separate the speckle fluctuations from the underlying SAR refectivity, a statistical modeling of speckle is necessary. In the overwhelming majority of cases, the speckle model assumes spatial independence from a pixel to the next. This assumption is valid only if the SAR image is sampled at the Shannon-Nyquist critical rate and no spectral apodization is applied. This is generally not the case of SAR images provided by spatial agencies. If only the intensity information is available, the spatial correlation of speckle can be reduced by sub-sampling the image. This however decreases the spatial resolution of the image. When the single-look complex (SLC) image is available, it is possible to decorrelate the speckle by carefully undoing the spectral apodization, the zero-padding and, in the case of Sentinel-1 TOPS acquisition mode, deramping and demodulating the images. Without spectral apodization, strong targets

This work was partially supported by the ANR MIRIAM project ANR14-CE27-0019. produce the typical extended cardinal sine signature. These targets can be extracted to improve the processing. In this paper, we show how speckle decorrelation and strong targets extraction can improve the performance of speckle reduction methods.

\section{DERAMPING, DEMODULATION AND COMPUTATION OF THE PSEUDO-RAW SENTINEL-1 IMAGES}

\subsection{Deramping and demodulation of a Sentinel-1 SLC image}

As explained in [1], the TOPS SLC products undergo a linear frequency modulation which is due to the steering of the antenna in azimuth during the acquisition process. Inverting this linear frequency modulation is necessary for performing subpixel operations such as interpolation and resampling. This operation is called deramping. In addition to deramping, it is also useful to perform a so-called $d e$ modulation, which consists in centering the support of the complex spectrum on $\mathrm{OHz}$. This operation roughly consists in the estimation of the Doppler centroid frequency, followed by a global translation of the complex spectrum.

Let $v: \Omega \rightarrow \mathbb{C}$ be a TOPS SLC image of size $M \times N$ and discrete domain $\Omega=I_{M} \times I_{N}$, noting $I_{K}=\{0, \ldots, K-1\}$. Applying deramping and demodulation to $v$ boils done to computing the image $u: \Omega \rightarrow \mathbb{C}$ such that, for any pixel location $(x, y)$, we have

$$
u(x, y)=v(x, y) \cdot \Phi(\tau(x), \eta(y)) \cdot \Psi(\tau(x), \eta(y))
$$

where $\Phi$ and $\Psi$ are called the deramping and demodulation functions respectively, $\tau(x)$ corresponds to the range time of the pixels located in the $x$-th column of the image, and $\eta(y)$ corresponds to the azimuth time of the pixels located in the $y$-th row of the image.

The computation of the deramping and demodulation functions relies on the metadata attached on the TOPS SLC product, following the procedure described in [1]. For the sake of completness, we describe the main steps of this procedure. For all $(\tau, \eta) \in \mathbb{R}^{2}$, functions $\Phi$ and $\Psi$ are defined by:

$$
\begin{aligned}
\Phi(\tau, \eta) & =\exp \left(-i \pi \frac{k_{a}(\tau) k_{s}}{k_{a}(\tau)-k_{s}} \cdot\left(\eta-\eta_{\mathrm{ref}}(\tau)\right)^{2}\right) \\
\Psi(\tau, \eta) & =\exp \left(-2 i \pi f_{\eta c}(\tau) \cdot\left(\eta-\eta_{\mathrm{ref}}(\tau)\right)\right) \\
\text { where } \quad k_{s} & =2 \frac{V_{s}}{c} f_{c} k_{\psi} \quad \text { and } \quad \eta_{\mathrm{ref}}(\tau)=\frac{f_{\eta c}(0)}{k_{a}(0)}-\frac{f_{\eta c}(\tau)}{k_{a}(\tau)}
\end{aligned}
$$

In the definition of $k_{s}$ above, $c$ denotes the speed of light (in $\mathrm{m} / \mathrm{s}$ ), $V_{s}$ the spacecraft velocity $(\mathrm{m} / \mathrm{s}), f_{c}$ the radar frequency $(\mathrm{Hz})$ and $k_{\psi}$ the antenna steering angle $(\mathrm{rad} / \mathrm{s})$. Both values of $f_{c}$ and $k_{\psi}$ can be 


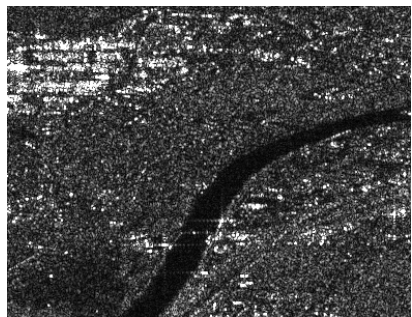

(a) modulus of a TOPS SLC image $v$

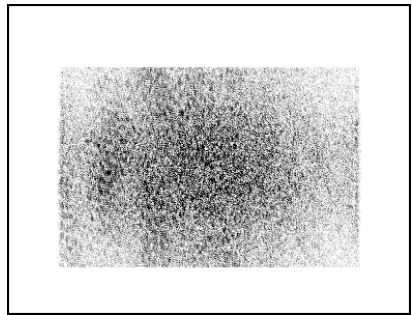

(c) Fourier spectrum after deramping

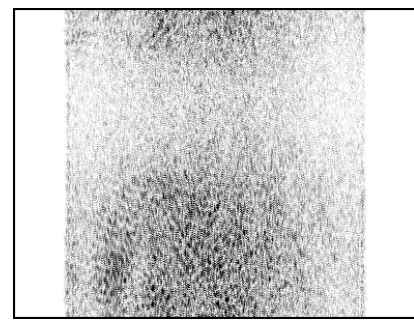

(b) Fourier spectrum of $v$ (bright $=$ low values, dark $=$ high values)

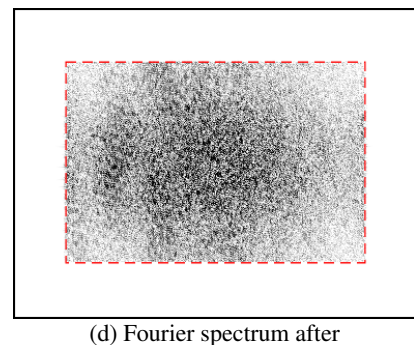

deramping and demodulation
Fig. 1: Deramping and demodulation of a TOPS Sentinel-1 SLC image. A TOPS SLC image $v$ whose modulus is displayed in (a) undergoes a linear frequency modulation which results in a spreading of the spectrum along the vertical direction (b). A pointwise multiplication in the spatial domain between $v$ and $\Phi$ leads to the deramped image whose spectrum has a rectangular support, as displayed in (c). Demodulation is done by multiplying the deramped image by $\Psi$, which centers the rectangular spectrum support on the zero-frequency, as displayed in (d). It is interesting to notice that deramping and demodulation operations do not change the modulus of the signal, but ony the phase information.

found explicitely in the metadata of the TOPS SLC product. Therefore, only $V_{s}, k_{a}(\tau)$ and $f_{\eta c}(\tau)$ need to be computed. The three components (in cartesian coordinates) of the spacecraft velocity can be found in the metadata for several azimuth times. Interpolating those values at the given azimuth time $\eta$ (e.g. using bilinear interpolation), we can estimate the spacecraft velocity. In the equations (2) and (3), the dependency of $V_{s}$ and $k_{s}$ on the variable $\eta$ is not made explicit by the notation, following the same convention as in [1]. The computation of $k_{a}(\tau)$ is performed based on the model

$$
k_{a}(\tau)=c_{0}+c_{1}\left(\tau-\tau_{0}\right)+c_{2}\left(\tau-\tau_{0}\right)^{2},
$$

where $\tau_{0}$ and the polynomial coefficients $c_{0}, c_{1}$ and $c_{2}$ are measured (and made available in the metadata) at several azimuth times. Like $k_{a}$, these coefficients are time dependent. For a given range time $\tau$, we can compute $k_{a}(\tau)$ at each tabulated azimuth time, and interpolate the resulting signal at the desired azimuth time $\eta$. Exactly the same approach is used to compute $f_{\eta c}(\tau)$ which also relies on a sequence of second order polynomials.

\subsection{Computation of a pseudo-raw Sentinel-1 image}

Let us consider from now the deramped and demodulated image $u$ defined in (1). Let $\widehat{u}$ denote the discrete Fourier transform (DFT) of $u$, defined by

$$
\forall(\alpha, \beta) \in \mathbb{Z}^{2}, \quad \widehat{u}(\alpha, \beta)=\sum_{(k, \ell) \in \Omega} u(k, \ell) e^{-2 i \pi\left(\frac{\alpha k}{M}+\frac{\beta \ell}{N}\right)} .
$$

As can be seen in Fig. 1 (d), the Fourier spectrum $\widehat{u}$ has a rectangular support $\widehat{\omega} \subsetneq \widehat{\Omega}$ (delimited by the red dashed-rectangle in Fig. 1 (d)), showing that the image $u$ has been sampled above the ShannonNyquist critical rate (oversampling). Besides, the spectrum $\widehat{u}$ also underwent some attenuation, more precisely, for all $(\alpha, \beta) \in \widehat{\Omega}$, we have

$$
\widehat{u}(\alpha, \beta)=\left\{\begin{array}{cl}
\widehat{u_{0}}(\alpha, \beta) \cdot \gamma(\alpha, \beta) & \text { if }(\alpha, \beta) \in \widehat{\omega} \\
0 & \text { otherwise }
\end{array}\right.
$$

where $\gamma: \widehat{\omega} \rightarrow \mathbb{R}_{++}$denotes the spectral weighting function (or apodization), and $u_{0}$ is called hereafter the pseudo-raw image. The pseudo-raw image corresponds to the image that would have been acquired at the Shannon-Nyquist critical sampling rate without any spectral weighting. The dimensions $m \times n$ of the frequency support $\widehat{\omega}=\widehat{I}_{m} \times \widehat{I}_{n}$, noting $\widehat{I}_{K}=[-K / 2, K / 2) \cap \mathbb{Z}$, can be obtained based on the bandwidth and sampling frequencies,

$$
m=\left\lfloor\frac{B_{\mathrm{r}}}{f_{\mathrm{r}}} \cdot M\right\rceil, \quad n=\left\lfloor\frac{B_{\mathrm{az}}}{f_{\mathrm{az}}} \cdot N\right\rceil,
$$

where $L \cdot\urcorner$ denotes the rounding function, $f_{\mathrm{r}}$ and $f_{\mathrm{az}}$ the sampling frequency in range and azimuth directions, and $B_{\mathrm{r}}$ and $B_{\mathrm{az}}$ the bandwidth in the corresponding directions, all available through the metadata of the TOPS SLC product. Thanks to the centering of the spectrum provided by the demodulation, we can automatically find the position of the frequency support $\widehat{\omega}$. Besides, we explained in [2] how the apodization function $\gamma$ could be estimated (if unknown), so that we can invert (6) and compute the pseudo-raw image $u_{0}$. An example of a pseudo-raw image $u_{0}$ computed from a TOPS SLC image $v$ is displayed in Fig. 2. Since the TOPS SLC image $v$ undergoes an important phase modulation due to the phase-ramping, this image cannot be directly interpolated using the standard Shannon interpolation. This is particularly visible in the left-hand side of Fig. 2 (c), where we display the Shannon interpolate of the image $v$ in the vicinity of a bright target, leading to unrealistic high frequency patterns in the azimuth direction. After deramping, we get an image which is compatible with Shannon interpolation and that can be easily manipulated at the subpixellic scale. As explained in [2, 3], computing the pseudo-raw image, such as that displayed in Fig. 2 (b), is particularly interesting from a statistical viewpoint, since the speckle in homogeneous regions exhibits almost no spatial correlation in contrast to the spatially correlated original image. Correlations in the original images are due to the oversampling and the spectral apodization. The pseudo-raw images also exhibit very strong sidelobes around bright targets (especially in urban areas) which is due to the cardinal sine response of those targets, as illustrated in the right side of Fig. 2 (c). In what follows, we illustrate how those targets can be efficiently handled via the subpixellic methods that we recently proposed in [3].

\section{BRIGHT TARGETS EXTRACTION AND RELOCALIZATION IN PSEUDO-RAW IMAGES}

The range and azimuth profiles of isolated bright targets in the pseudo-raw images match very well cardinal sine functions, as illustrated in the right side of Fig. 2 (c). Therefore, the contribution of a bright target to the pseudo-raw image can be modeled by

$$
\forall(k, \ell) \in \omega, \quad u_{0}(k, \ell)=A \operatorname{sinc}(k-x, \ell-y)+u_{0}^{*}(k, \ell),
$$

where $A \in \mathbb{C}$ denotes the complex amplitude of the bright target, $(x, y) \in[0, m) \times[0, n)$ the subpixellic position of its center, 


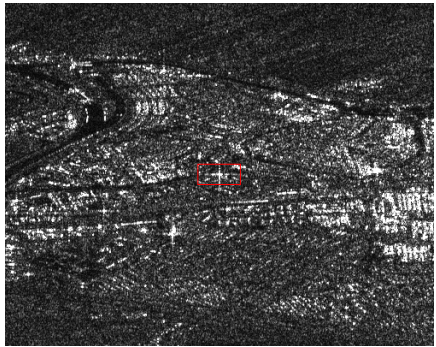

(a) TOPS SLC image $v$

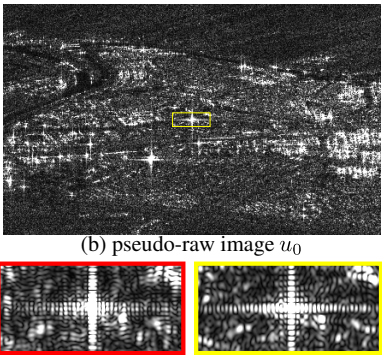

(c) Shannon interpolation of bright targets

Fig. 2: Pseudo-raw Sentinel-1 images. We display in (a) the modulus of a TOPS SLC image $v$, and in (b) the modulus of the pseudoraw image $u_{0}$ computed from $v$. Both images exhibit different pixel sizes because of the resampling involved in the computation of $u_{0}$ to remove the zero-padding.

$\operatorname{sinc}(s, t)=\sin (\pi s) /(\pi s) \cdot \sin (\pi t) /(\pi t)$ the 2D-separable product of cardinal sine functions, and $u_{0}^{*}$ the pseudo-raw image that we would have observed in the absence of the target. We recently proposed in [3] an algorithm for the detection and the extraction of bright targets with cardinal sine profile such as in (8). We apply in this paper the algorithm to Sentinel-1 pseudo-raw images $u_{0}$. In practice, the algorithm returns a set $\mathscr{C}=\left\{\left(x_{j}, y_{j}, A_{j}\right)\right\}_{1 \leq j \leq T}$ where $T$ represents the number of meaningful targets (automatically derived by the algorithm thanks to an a contrario criterion), and such that the $j$-th target is characterized by its subpixellic position $\left(x_{j}, y_{j}\right) \in[0, m) \times[0, n)$ and its complex amplitude $A_{j} \in \mathbb{C}$. After detection of the targets, we can form a decomposition of the image $u_{0}$ into the sum of a target component, noted $\mathcal{S}_{0}(\mathscr{C})$, which is the linear combination of cardinal sine functions defined by

$$
\forall(k, \ell) \in \omega, \quad \mathcal{S}_{0}(\mathscr{C})(k, \ell)=\sum_{j=1}^{T} A_{j} \operatorname{sinc}\left(k-x_{j}, \ell-y_{j}\right),
$$

and a speckle component $w_{0}=u_{0}-\mathcal{S}_{0}(\mathscr{C})$, which represents the image that would have been acquired in the absence of the targets of $\mathscr{C}$. An example of such decomposition of a Sentinel-1 pseudoraw image is displayed in Fig. 3. As suggested in [3], an interesting way to suppress the sidelobes consists in recombining the extracted targets as a linear combination of discrete Diracs, which corresponds to computing the image $R_{\omega}\left(u_{0}\right)=w_{0}+\mathscr{D}_{\omega}(\mathscr{C})$, noting $D_{\omega}(\mathscr{C})=\sum_{j=1}^{T} A_{j} \delta_{\left\lfloor x_{j}\right\rceil,\left\lfloor y_{j}\right\rceil}$, and $\delta_{(k, \ell)}$ the discrete Dirac centered at $(k, \ell)$ (taking the value 0 everywhere except at position $(k, \ell)$ where it takes the value 1$)$. An example of such recombined image is displayed in Fig. 3 (b). Beyond the interesting sidelobes suppression offered by this approach, we illustrate in the next section how such a decomposition can improve the quality of speckle reduction methods.

\section{IMPACT OF RESAMPLING AND TARGET EXTRACTION ON SPECKLE FILTERING}

With the short revisit time of TerraSAR-X and Sentinel-1 satellite constellations, long time series can be obtained. These SAR images can then be combined in order to produce images with strongly suppressed speckle while preserving the spatial resolution. The recent RABASAR framework [4] offers a simple yet surprisingly efficient way to exploit the temporal information: a so-called superimage is produced by combining temporal multi-looking and an ad-

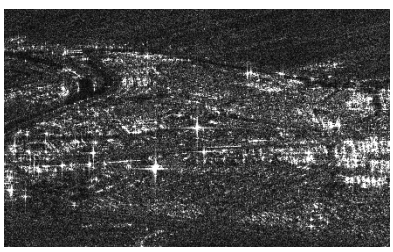

(a) pseudo-raw image $u_{0}$

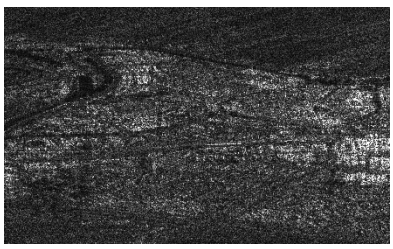

(c) speckle component $w_{0}$

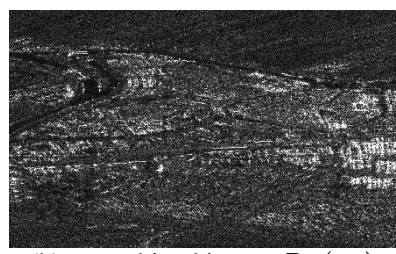

(b) recombined image $R_{\omega}\left(u_{0}\right)$

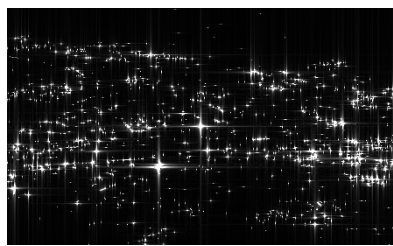

(d) target component $\mathcal{S}_{0}(\mathscr{C})$

Fig. 3: Speckle plus target decomposition of Sentinel-1 images. We display in (a) the modulus of a pseudo-raw image $u_{0}$, in (c) the modulus of its speckle component $w_{0}$ and in (d) the modulus of its target component $\mathcal{S}_{0}(\mathscr{C})$. The set of targets $\mathscr{C}$ extracted from $u_{0}$ was computed using the decomposition algorithm proposed in [3]. By construction, we have $u_{0}=w_{0}+\mathcal{S}_{0}(\mathscr{C})$. Replacing the linear combination of cardinal sines $\mathcal{S}_{0}(\mathscr{C})$ by a linear combination of discrete Diracs $\mathscr{D}_{\omega}(\mathscr{C})$ in this additive decomposition yields the image $R_{\omega}\left(u_{0}\right)$, whose modulus is displayed in (b): this image is free of sidelobe effects.

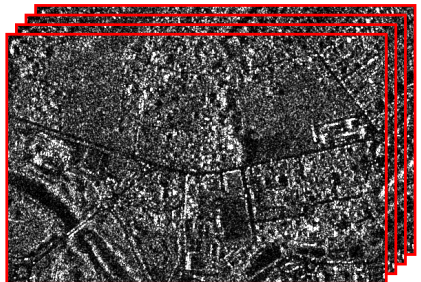

(a) stack of SLC images

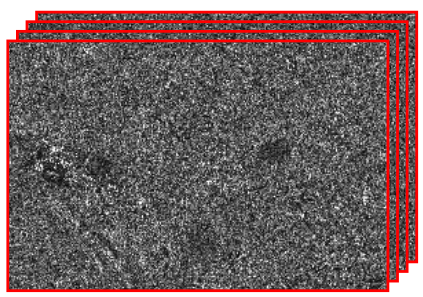

(c) stack of (subsampled) ratios

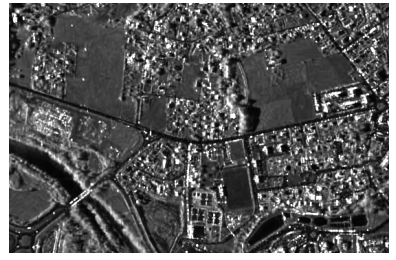

(b) super-image

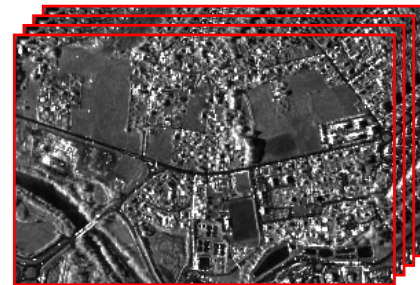

(d) stack of denoised images
Fig. 4: Principle of multi-temporal speckle reduction with RABASAR. Temporal multi-looking of a stack of SLC images (a) and an additional speckle-reduction step produce a high signal-tonoise ratio image called the super-image (b). Dividing the stack (a) by the super-image (b) gives a stack of ratio images (c). Timespecific changes are visible in these ratio images. After speckle reduction of the ratio images and recombination with the super-image, the final stack of restored images is obtained (d). One can notice that the stack of denoised images (d) is more faithful to the stack of SLC images (a) than the super-image (b). However, in this framework, subsampling of the ratio images (c) is necessary to avoid restoration artifacts caused by strong speckle correlations.

ditional spatial speckle-reduction step designed to remove the residual speckle fluctuations. This super-image contains most of the spatial structures that are present in an image at any given date $t$ (roads, field boundaries, buildings, forests), but at a much improved signal- 


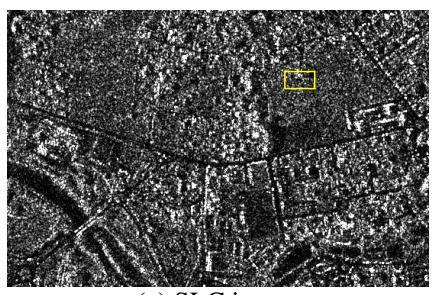

(a) SLC image

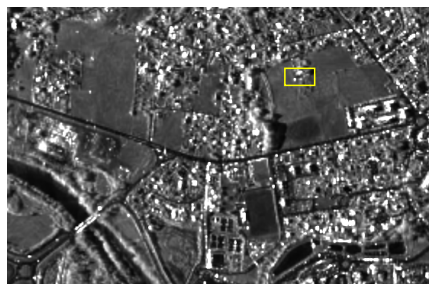

(c) RABASAR denoising of (a)

using subsampling of factor 2

Fig. 5: Denoising a stack of TerraSAR-X SLC images. We used RABASAR to denoise a stack of 20 SLC images. We display in (a) one of the images of this stack. On the one hand, denoising (a) without subsampling the intermediate ratio image yields the image (b) with artifacts in homogeneous areas, due to the spatial correlations of the speckle. One the other hand, using subsampling reduces those artifacts but affects the image quality: we observe in (c) a loss of details and some aliasing artifacts. Besides, in both situations (b) and (c), we can observe that targets that were not present in the initial image appear in the denoised image (e.g. in the yellow rectangle). Noting $u_{0}$ the pseudo-raw image associated to (a), we display in (d) the denoising of $R_{\omega}\left(u_{0}\right)$ obtained using the super-image computed from the speckle-components $w_{0}$ of the whole stack. Image (d) is free of the artifacts observed in (b) and (c).

to-noise ratio, as illustrated in Fig. 4 (b). However, the reflectivity of the scene varies from one date to another and some abrupt changes may occur. In order to produce a despeckled image that is faithful to the content of the image at date $t$, the ratio image between the observation at date $t$ and the super-image is formed. This ratio is filtered using a single-image speckle reduction algorithm. Should the super-image perfectly match the reflectivity of the scene at date $t$, the ratio image will contain pure (stationary) speckle noise. On the converse, areas where the reflectivity at date $t$ differs from the superimage will appear in the ratio image as a speckle with a mean value that differs from 1. After despeckling this ratio image, the filtered image at date $t$ is obtained by multiplication with the super-image, as illustrated in Fig. 4 (d). See [4] for more details on the method.

In RABASAR framework, two speckle-reduction steps are performed: one to obtain the super-image, the other to filter the ratio image. In each of these two steps, spatial correlation of the speckle is an issue. In practice, images are down-sampled to reduce speckle correlation, which causes a resolution loss. This is illustrated in Fig. 5 (TerraSAR-X) and Fig. 6 (Sentinel-1), where we can see that, without subsampling prior to despeckling, the denoised image (b) exhibits some strong artefacts in homogeneous areas, while, when subsampling is used, the artefacts are attenuated at the cost of a severe loss of resolution in the denoised image (c) and even aliasing artifacts (visible in Fig. 5). Another issue in the multi-temporal filtering by RABASAR is that some bright targets, present in the super-image but not in a given SLC image at time $t$, may appear when multiplying the denoised ratio by the super-image, at the end

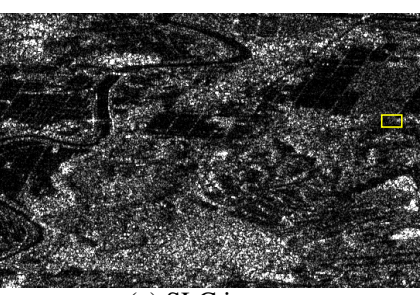

(a) SLC image

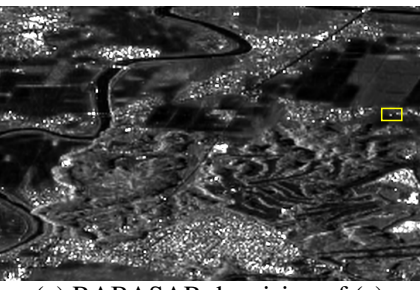

(c) RABASAR denoising of (a) using subsampling of factor 2

Fig. 6: Denoising a stack of Sentinel-1 images. We performed the same experiment as in Fig. 5 on a stack of Sentinel-1 SLC images. The restoration (b) displays the same artifacts in homogeneous areas as observed in Fig. 5. Those artefacts are avoided in (c), thanks to subsampling before despeckling, but also in (d), thanks to the speckle plus target decomposition. Restoration (d) has a slightly improved resolution compared to (b) and also avoids phantom targets such as that observed in the yellow rectangle. However, the differences between those two restoration is more modest than in the case of the TerraSAR-X images of Fig. 5. It seems to be harder, at the spatial resolution of Sentinel-1, to preserve fine details during the MuLoG denoising step involved in RABASAR.

of the process. This is illustrated in Fig. 5 and Fig. 6, where we indicate with a yellow rectangle, the presence of a phantom target that is present (b) and (c), but absent in the initial SLC image (a). Thanks to the speckle plus target decomposition described in Section 3, we are able to replace each pseudo-raw SLC image $u_{0}$ of the stack by a SLC image $R_{\omega}\left(u_{0}\right)=w_{0}+\mathscr{D}_{\omega}(\mathscr{C})$, where the speckle component $w_{0}$ has no spatial speckle correlations in homogeneous areas and $\mathscr{D}_{\omega}(\mathscr{C})$ is a linear combination of discrete Diracs. Besides, computing the super-image only using the stack of speckle components $w_{0}$ yields an image without bright targets. Therefore, applying the RABASAR framework to denoise a SLC image $R_{\omega}\left(u_{0}\right)$ using such target-free super-image prevents the aforementioned phantom target phenomenon. Besides, the ratio between $R_{\omega}\left(u_{0}\right)$ and the superimage being uncorrelated, it can be efficiently denoised, as we show in Fig. 5 (d) and Fig. 6 (d).

\section{REFERENCES}

[1] N. Miranda, "Definition of the TOPS SLC deramping function for products generated by the S-1 IPF," Tech. Rep., ESA, 2014.

[2] R. Abergel, S. Ladjal, F. Tupin, and J. Nicolas, "A complex spectrum based SAR image resampling method with restricted target sidelobes and statistics preservation," in IGARSS, 2017.

[3] R. Abergel, L. Denis, S. Ladjal, and F. Tupin, "Subpixellic methods for sidelobes suppression and strong targets extraction in single look complex SAR images," IEEE JSTARS, 2018.

[4] W. Zhao, C. Deledalle, L. Denis, H. Maitre, J. Nicolas, and F. Tupin, "RABASAR: A fast ratio based multi-temporal SAR despeckling," in IGARSS, 2018. 